In der Rubrik „Literatur kompakt" werden die wichtigsten Originalarbeiten aus der internationalen Fachliteratur referiert.

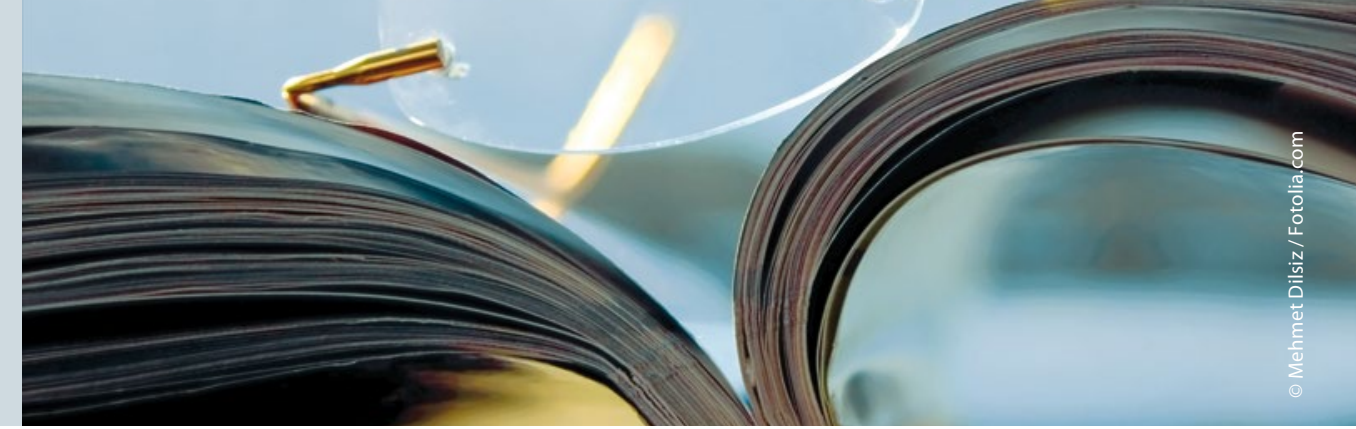

\section{Fertilitätserhaltende Therapie des frühen Endometriumkarzinoms möglich}

Etwa 7\% der Patientinnen mit Endometriumkarzinom sind unter 45 Jahre alt. Bei lokal begrenztem, niedriggradigem Tumor ist eine Hormontherapie eine fertilitätserhaltende Behandlungsoption. Allerdings fehlten bisher Daten zur Sicherheit dieses Vorgehens in Bezug auf das Langzeitüberleben.

U m hierzu mehr Sicherheit zu erhalten, analysierten Forscher um Zoë R. Greenwald Daten aus der US-amerikanischen SEER(Surveillance, Epidemiology, and End Results)-Datenbank. Aufgenommen wurden 6.339 unter 45-jährige Frauen, die zwischen 1993 und 2012 wegen eines lokal begrenzten, niedriggradigen Endometriumkarzinoms behandelt worden waren. Davon hatten 161 primär eine Hormontherapie (HT) erhalten, 6.178 waren primär operiert worden. Um vergleichbare Gruppen zu erhalten, wendeten die Forscher ein Propensity-Score-Matching an.

Adjustiert im Hinblick auf die Faktoren Alter, Diagnosezeitpunkt, Familienstand, Herkunft, Tumorgrad, Morphologie und vorangegangene Strahlentherapie ergab sich nach einer Beobachtungszeit von 15 Jahren kein Unterschied hinsichtlich der Gesamtmortalität. Sie lag in der HT-Gruppe bei $14,1 \%$ (95\%-Konfidenzintervall [95\%-KI] 6,7$28,4 \%)$ und in der anhand des Propensity-Score abgeglichenen Gruppe mit chirurgischer Therapie bei 9,3\% (95\%-KI 4,1-20,5\%). Die krebsspezifische Mortalität nach 15 Jahren war nach der primären Analyse allerdings bei HT leicht erhöht, mit 9,2\% (95\%-KI 3,4$24,0 \%$ ) gegenüber $2,1 \%$ in der Gruppe mit chirurgischer Therapie (95\%-KI
1,5-2,8\%). Diese Differenz basierte aber hauptsächlich auf nur drei späten Todesfällen. Eine Sensitivitätsanalyse mit einer breiteren Definition der krebsspezifischen Mortalität ergab keinen Unterschied zwischen den Behandlungsgruppen mehr. Das Sterberisiko insgesamt war nicht signifikant erhöht (Hazard Ratio 1,45 ; $95 \%$-KI 0,44-4,74).

Die Forscher plädieren für die Einrichtung einer zentralen Datenbank für die fertilitätserhaltende Hormontherapie beim frühen Endometriumkarzinom.

Basierend auf dieser retrospektiven, populationsbasierten Analyse ist das Überleben jüngerer Frauen bei niedriggradigem Endometriumkarzinom also sehr gut - unabhängig von der Primärtherapie. Bei entsprechend enger Selektion kann ihnen nach Meinung der Forscher eine HT zum Fertilitätserhalt angeboten werden, wenn eine engmaschige Beobachtung durch erfahrene Kliniker gewährleistet ist. Nach Abschluss der Familienplanung sollte dann umgehend die Hysterektomie erfolgen.

Friederike Klein

Greenwald ZR et al. Does hormonal therapy for fertility preservation affect the survival of young women with early-stage endometrial cancer? Cancer 2017;123(9):1545-54

\section{Kommentar}

Die vorgelegte Studie bestätigt in eindrucksvoller Weise die Empfehlungen der bislang gültigen Leitlinien zur Therapie des frühen Endometriumkarzinoms. Auch wenn die Gruppe der organerhaltend mit einer HT behandelten Frauen mit frühem Endometriumkarzinom mit 161 vergleichsweise gering ist, so zeigen diese Daten doch, dass durch ein organerhaltendes Vorgehen mit Verzicht auf eine primäre Radikaloperation bei einem frühen Endometriumkarzinom das Gesamtüberleben nicht signifikant reduziert wird. Die Beobachtung von drei späten Todesfällen bestätigt jedoch ebenfalls die Empfehlungen der Leitlinie, dass nach Abschluss der Familienplanung auch bei bislang unauffälligem Verlauf unbedingt in allen Fällen eine sekundäre Hysterektomie erfolgen muss. Eine fertilitätserhaltende HT des frühen Endometriumkarzinoms ist somit onkologisch verantwortbar, es sollte jedoch unbedingt darauf hingewiesen werden, dass ein organerhaltendes Vorgehen nur mit dem Ziel einer Schwangerschaft erfolgen sollte. Da das Auftreten eines Endometriumkarzinoms aufgrund der bekannten Risikofaktoren überdurchschnittlich häufig auch mit einer Infertilität verbunden ist, soll allen Frauen im Anschluss an die histologische Diagnose bei Entschluss zu einem organerhaltenden Vorgehen unbedingt zu einer Kinderwunschbehandlung nach unauffälliger Kontrollabrasio geraten werden.

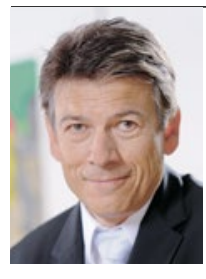

Prof. Dr. med. Peter Mallmann

Klinik und Poliklinik für Frauenheilkunde und Geburtshilfe, Uniklinik Köln peter.mallmann@ uk-koeln.de 